\title{
A SURVEY IDENTIFYING TRENDS ON USE OF SOFTWARE DEVELOPMENT TOOLS IN DIFFERENT INDIAN SMES
}

\author{
Nomi Baruah ${ }^{1}$ and Ashima ${ }^{2}$ \\ ${ }^{1}$ Computer Science and Engineering Department, Thapar University, Patiala, India \\ baruahnomiegmail.com \\ ${ }^{2}$ Computer Science and Engineering Department, Thapar University, Patiala, India \\ ashima@thapar.edu
}

\begin{abstract}
Software Process Improvement defines the identification of the current state-of- practice of processes within an organization and then improving it. Software Process Improvement is ever lasting, never ending and ever changing process. Some of the issues which force an organization to undergo software process improvement are customer dissatisfaction, inadequate software quality, inability to deliver on time and within budget, and excessive rework. The SMEs are using software process models but they are not able to deliver on time and within budget, and excessive rework. The SMEs are using software process improvement models but they are not able to follow all the processes due to lack of resource and cost to improve their productivity and quality of their product. A survey of 18 SMEs catering software market has been carried out for finding software development scenarios.The intent of the study was to find the prevailing tools and techniques, the SMEs are using to automate software development process and to incorporate software project management. The survey identifies four different types of Software Development Tools which are proving to be effective in current scenario of software development. These are identified as Requirement Management Tools, Process Modelling Tools, Software Configuration Management Tools and Cost Estimating Tools. This paper summarizes the trends followed in usage of Software Development Tools in SMEs and it has been shown graphically also.
\end{abstract}

\section{KEYWORDS}

Software Development Tools,Requirement Management Tool,Process Modeling Tool,Configuration Management Tools and Cost Estimation Models

\section{INTRODUCTION}

The small and medium enterprises are striving impatiently to improve their productivity and quality with continuous changing market demands. The software development is expanding multidimensionally in different fields.So,they are pursuing to improve their software processes. Software development tools help the project teams to address quality, cost, schedule, performance and supportability so that they can determine software program success and failure [24].

\section{SOFTWARE DEVELOPMENT TOOLS}

Software Development Tool is a program or application that software developers used to create, debug, maintain or otherwise support other programs and application [16].

DOI : $10.5121 /$ ijsea.2012.3503 


\section{Www. Requirement Management Tools}

Requirement Management Tools assist an organization in defining and documenting requirements by allowing them to store requirements in a central location. Project teams can then access the requirements in a central location. Project teams can then access the requirements by allowing them to ensure that their needs were needs correctly specified[26].

\subsection{Aaa. Rational Suite Analyst Studio}

The Rational Suite Analyst Studio is a requirement management tool used by business and system analysts responsible for gathering and managing software requirements. The tool allows reporting and tracking enhancement requests and defects in a powerful change request management system shared by the entire team and it also integrates data from multiple sources throughout the project [14].

\subsection{Bbb.Omni-Vista OnYour Mark Pro}

Omni-Vista OnYour Mark Pro is a requirement management tool which allows users to do intelligent trade-off analysis between requirements, schedules and development costs. Users enter the difficulty of each requirement [12].

\subsection{Ccc. Project Management Tool}

Project Management Tool is about taking ideas and converting them into a planned, resourced and funded project. The project management process can be broken down into three stages: Project Initiation, Project Control and Project Closure [15].

\subsection{Ddd. Bugzilla}

Bugzilla is a web-based tool that lets users report and look up existing bugs. Changes made to a bug's status are automatically sent to users concerned with it [7].

\subsection{Eee.Redmine}

Redmine is a flexible project management web application which is written using the the Ruby on Rails framework. It has cross-platform and cross-database. Redmine is an open source and is released under the terms of the GNU General Public License v2 [18]

\section{Xxx.Process Modeling Tools}

Process Modeling Tools are complex and powerful analysis tools for corporate process library, process analysis and organization architecture [25].

\subsection{Aaa.BizAgi Process Modeler}

BizAgi Process Modeler is a freeware business process modeling and documentation tool. The modeler enables to visualize diagram, model and document business processes in industry standard Business Process Model and Notation [28]. 


\subsection{Bbb.Open ModelSphere}

Open ModelSphere is an integrated tool for business process,data and UML modeling. The Open ModelSphere is used for building whole or partial models of an organization from the viewpoint of the process analyst the database designer or object developer [16].

\subsection{Ccc.Questra BPM Suite}

Questra BPM Suite is a web-based business management system. The monitoring function enables tracking and measuring employee performance on the cloud [11].

\subsection{Ddd.Tibco Business Studio}

Tibco Business Studio is used for business analyst and solution designer including those responsible for the analysis, design, simulation, implementation and deployment of business processes [27].

\subsection{Eee. Process Maker}

Process Maker is a cost effective and easy to use open source business process management which can assist organization of any size with designing, automating and deploying business processes of various kinds [17].

\section{Yyy.Software Configuration Management Tool}

Software Configuration Management Tool enables the project managers to keep evolving software product under control and thus contributes to satisfying quality and delay constraints [23].

\subsection{Aaa.GNU Arch}

GNU Arch manipulate changes made by multiple people or at different times [8].GNU Arch is a software configuration tool used to keep track of the changes made to a source tree and to help programmers .

\subsection{Bbb. Rational ClearCase}

Rational ClearCase is a software tool for software configuration management of source code and other software development assets. It forms the base of version control for many large and medium sized businesses and can handle projects with hundreds or thousands of developers [22].

\subsection{Ccc.Concurrent Version System}

Concurrent Version System is a SCM tool for software developers which support collaborative development of software development of software development of software within a team,and the tracking of changes to software source code over time. The tool supports latest changes to the software [10]. 
International Journal of Software Engineering \& Applications (IJSEA), Vol.3, No.5, September 2012

\subsection{Ddd.Serena ChangeMan DS}

Serena ChangeMan DS features a full suite of SCM capabilities,from versioning through build and deployment , all based on user-defined processes.It manages distributed software application from development to development .It supports the most common operating systems using a client-server and agent based architecture [21].

\subsection{Eee.SubVersion}

SubVersion is a version control system that keeps track of changes made to files and folders ,facilitating data recovery and providing a history of the changes that have been made over time [9].

\section{Zzz. Cost Estimation Models}

Cost Estimation Model calculates the approximate cost of a product, program or project computed on the basis of the available information [13].

\subsection{Aaa.Advanced Missions Cost Model}

Advanced Missions Cost Model is a simple online method that provides for a quick turnaround and rough-order-of-magnitude estimating. The model is used for estimating the development and production cost of spacecraft, space transportation systems, aircraft, missiles, ships and land vehicles [2].

\subsection{Bbb.Cost Estimating Guidelines}

The Cost Estimating Guidelines provides Information and Information Technology (I \& IT) project managers, project analysts and PMO staff with an understanding of the project cost estimates recommended at each phase of the I \& IT project life cycle [1].

\subsection{Ccc.COCOMO Software Cost Model}

COCOMO stands for Constructive Cost Model.COCOMO is an open-model [19].It is used for estimating the number of person-months required to develop software. It estimates the development schedule in months and produces an effort and schedule distribution by major phases[4].

\section{4.Ddd.Cost Spreading Calculator}

Cost Spreading Calculator used to estimate the cost of a program upto 8 years. A beta curve is used by the calculator to determine the amount of money to be spent in each year based on the fraction of the total time that has elapsed. The user enters the total cost to be spread, the beginning and ending years [3].

\section{5.Eee.Learning Curve Calculator}

Learning Curve Calculator have been applied to all types of work from simple tasks to complex to complex jobs .It recognizes the repetition of the same operation results in less time or effort expended on that operation .It states that the direct labor man-hours necessary to complete a unit of production will decrease by a constant percentage each time the production quantity is doubled [5]. 


\section{SuRVey OF THE USE OF SOFTWARE DEVElopMent TOOLS}

A survey is carried on the use of Software Development Tools in 18 SMEs of India.They are FutureSoft,Delhi,IT Pyramid,Noida,Zaloni Technologies,Guwahati, DZ Engineering,Pune,Aon Hewitt,Chennai,CGI Technologies,Bangalore,IAG Automation,Gurgaon,SIQUES,Noida,Vacpic Technologies Pvt Ltd,Bangalore,Aris Global Software Pvt Ltd,Bangalore,Yodlee Pvt Ltd,Bangalore,Roma Think Soft,Bangalore,Exilant Technologies Pvt Ltd,Bangalore,Xixom Technology,Delhi,IQ Systems India Pvt Ltd,Chennai,Globrin Technologies,Gurgaon,Targus Technologies, Gurgaon and Verchaska Infotech Pvt Ltd,Mumbai.It has also been seen that though the SMEs are not using any proper SPI models, they are using different Software Development Tools in their organization to improve their productivity and quality.The different Software Development Tools are Requirement Management Tools, Software Project Planning Tools, Process Modeling Tools, Software Configuration Management Tools and Cost Models. A questionnaires concentrating rigorously on software process requirement engineering, software process models, software teams, software metrics, software planning and development, software process improvement models and software development documentation. The questions were filled up by Software Developers, Software Project Managers, Software Product Managers and Project Leaders. The different figures below show the percentage of use different Software Development Tools in Indian SMEs.

\section{1 Www. Percentage of Use of Requirement Management Tools in SMEs of India}

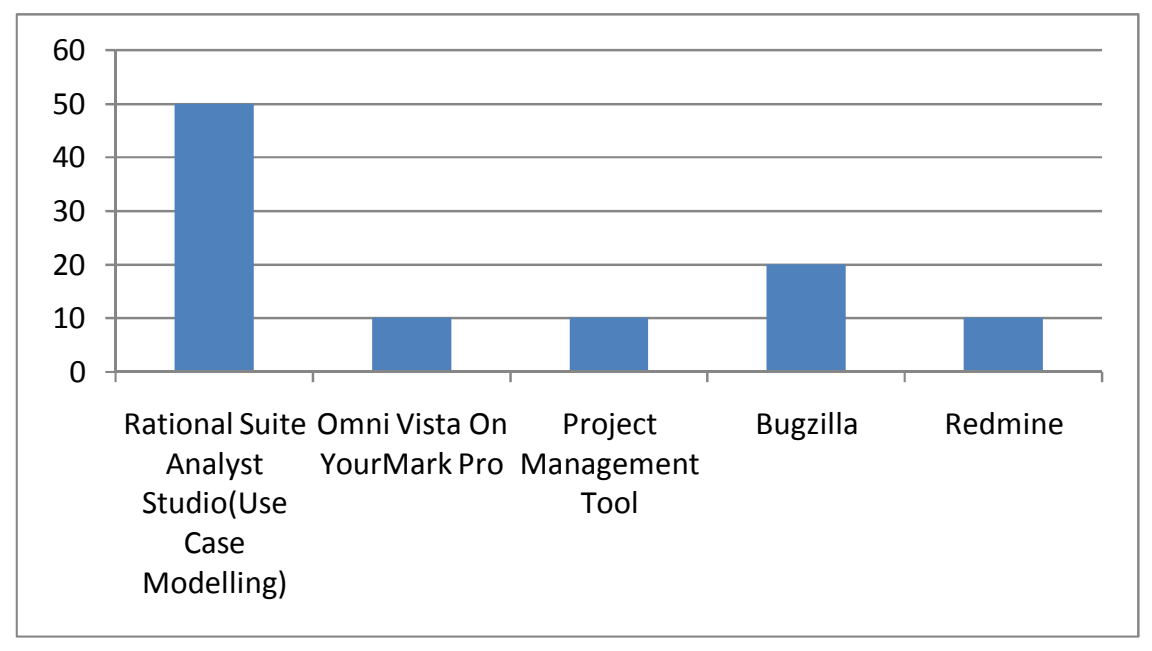

Figure 1. Percentage of Use of Requirement Management Tools in SMEs of India

It has been seen in Fig 1 most of the Indian SMEs uses Rational Suite Analyst Studio (Use Case Modelling) as requirement management tool in their organization while the other requirement tools are being used by different SMEs on small scale. 
International Journal of Software Engineering \& Applications (IJSEA), Vol.3, No.5, September 2012

\section{2 Xxx. Percentage of Use of Process Modeling Tools in SMEs of India}

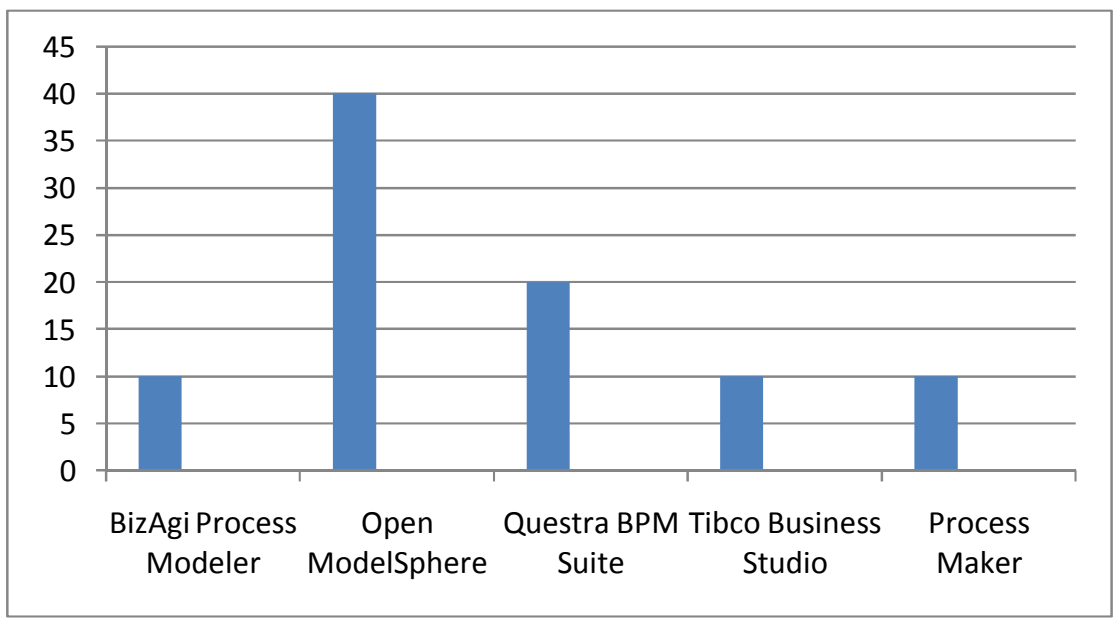

Figure 2. Percentage of Use of Process Modeling Tools in SMEs of India

It has been seen from Fig 2 that most of the Indian SMEs uses Open ModelSphere as Software Project Modeling Tool, while the other Process Modeling Tools are liked by few SMEs of India.

\subsection{Yyy. Percentage of Use of Software Configuration Management Tools in SMEs of India}

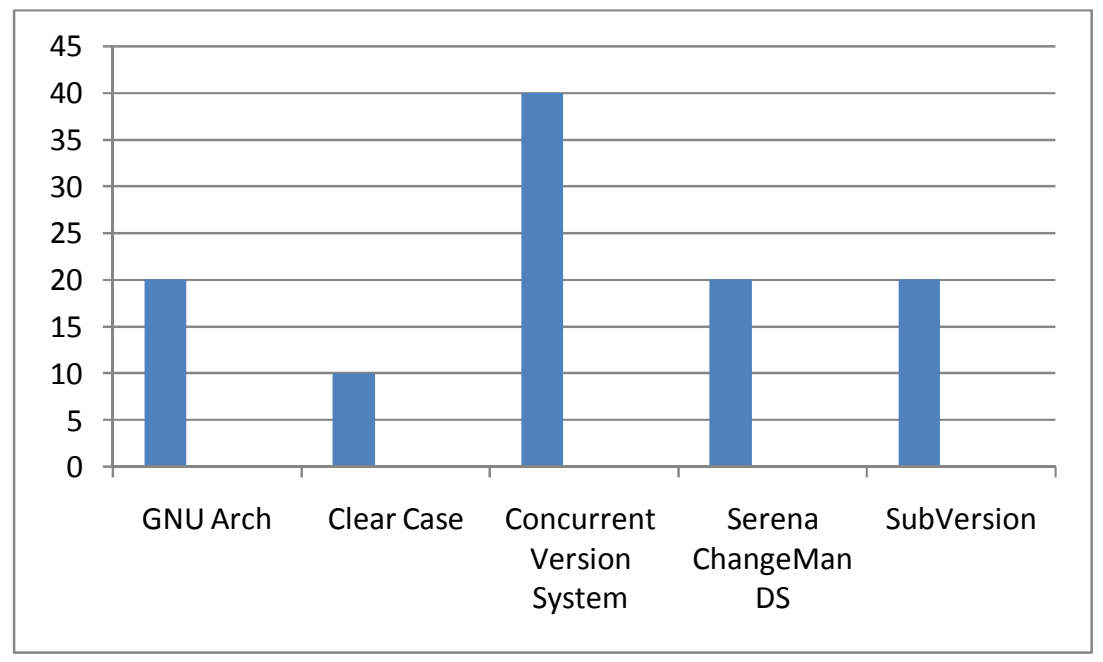

Figure 3. Percentage of Use of Software Configuration Management Tools in SMEs of India

It has been seen in Fig 3 that most of the Indian SMEs uses Concurrent Version System as Software Configuration Management Tools, while the other Software Configuration Management Tools like GNU Arch, Clear Case etc are being used by different SMEs of India. 


\subsection{Zzz. Percentage of Use of Cost Models in SMEs of India}

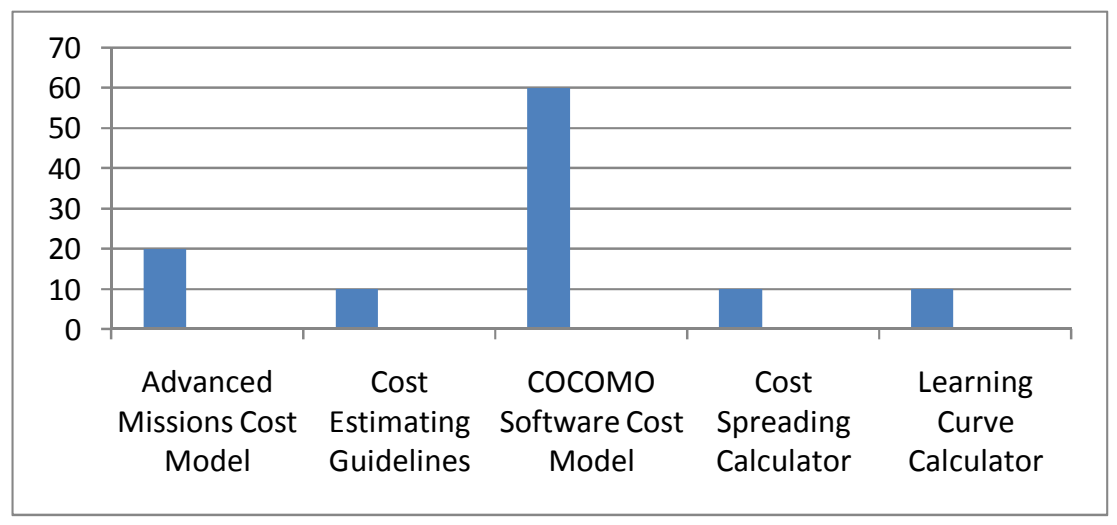

Figure 4. Percentage of Use of Cost Models in SMEs of India

It has been seen in Fig 4 that most of the Indian SMEs uses COCOMO Software Cost Model as Cost Model, while the other Cost Model like Advanced Missions Cost Model, Cost Estimating Guidelines etc are being used by different SMEs of India.

\section{Conclusion}

Software Development is a major activity that has to be carried out by software engineers, and project manager. SMEs in Indian Software Industry are bundled up with many outsourced national and international projects. Many of the SMEs are not following any software process improvement model to enhance their productivity and quality due of lack of resources. But Indian SMEs are quite capable of fulfilling the needs of software industry besides the fact of not following any particular software improvement model. To bridge the gap, these SMEs have adopted certain automated software development tools, which they are using effectively in order to deliver the product. A proper use of software development tools also reduce risks associated with the project and also promises a timely deliver. On the other hand, Use of Software Development tools like Requirement Management Tools,Process Modeling Tools,Software Configuration Management Tools and Cost Models are helpful in software project management also.

\section{ACKNOWLEDGEMENTS}

The authors would like to thank everyone i.e. Software Developers, Software Project Managers, Software Product Managers and Project Leaders of the above mentioned companies who had helped by filling the survey questionnaires, which had helped us in knowing the different strategies followed by different companies.

\section{REFERENCES}

[1] Cost Estimation Guidelines How to Develop Accurate Cost Estimates as Part of the Gateway Review Process, Vers 0.2,June 14,2012

[2] http://cost.jsc.nasa.gov/AMCM.html ,Feb 18,2012

[3] http://cost.jsc.nasa.gov/beta.html ,Feb 18,2012.

[4] http://cost.jsc.nasa.gov/COCOMO.html ,Feb 24,2012.

[5] http://cost.jsc.nasa.gov/learn.html ,Feb 24,2012. 
International Journal of Software Engineering \& Applications (IJSEA), Vol.3, No.5, September 2012

[6] http://en.wikipedia.org/wiki/Software_development_tools ,Feb 16,2012.

[7] http://linux.about.com/cs/linux101/g/bugzilla.htm, Feb 24,2012.

[8] http://savannah.gnu.org/projects/gnu-arch ,Feb 24,2012

[9] http://searchenterpriselinux.techtarget.com/definition/Subversion ,Feb 24,2012.

[10] http://sourceforge.net/apps/trac/sourceforge/wiki/CVS ,Feb 24,2012.

[11] http://store.questetra.com/en/ ,Feb 24,2012.

[12] http://www.atwebo.com/case.htm ,Feb 24,2012.

[13] http://www.businessdictionary.com/definition/cost-estimate.html ,Feb 24,2012.

[14] http://www.cdrummond.qc.ca/cegep/informat/Professeurs/diane/hiver/420-401/ Rational $\% \quad 20$ Rose/html/analysisstudio.html,Feb 24,2012.

[15] http://www.inc.com/guides/201102/20-essential-tools-for-project-management.html, Feb 24, 2012.

[16] http://www.modelsphere.org/Developer\%20Guide.pdf Feb 24,2012.

[17] http://www.processmaker.com/,Feb 24,2012.

[18] http://www.redmine.org/ June10,2012.

[19] http://www.softstarsystems.com/overview.htm ,Feb 24,2012.

[21] http://www.tandemworld.net/serena.html, Feb 24,2012.

[22] http://www.websters-online-dictionary.org/definitions/ClearCase ,Feb 24,2012.

[23] J. Estublier, Software Configuration Management: A Roadmap. Dassault Systemes SR, Grenoble University,France, 2000.

[24] L.H. Werth ,'Introduction to Software Process Improvement", Carnegie Mellon University,CMU/SEI-93-EM-8,1993.

[25] Process Modeling Tools- Comparison and Decision www.smartcities.info/Smart\%20Cities\%20\%20ProcessModellingTools[1].pdf ,Feb 24,2012.

[26] R.R. Sud and J.D. Arthur, Requirement Management Tools A Qualitative Assessment.Department of Computer Science,Virginia Tech,Blacksburg,VA 24060 USA,2002.

[27] TIBCO Business Studio ${ }^{\text {TM }}$ Process Modeling User's Guide, Software Release 3.5.3, March 2012, of Computer Science,Virginia Tech,Blacksburg,VA 24060 USA,2002.

[28] www.bizagi.com/.../BizAgi\%20Functional\%20Descr... - United Kingdom,Feb 24,2012.

\section{Author}

Nomi Baruah is a student.She had completed her Master of Engineering in Software Engineering from Thapar University,Patiala,Punjab in July,2012.Her research interest include Software Process Reengineering,Agile Software Development,Software Quality Improvement in small and medium enterprises and Unified Modeling Language.

Ashima is Assistant Professor in Computer Science and Engineering Department ,Thap-ar University,Patiala,India;and pursuing Ph.D in Computer Science from Faculty of Eng-ineering,UCOE,Punjabi University,Patiala,India.She holds a Bachelor of Technology (B.Tech) degree in Computer Science and Engineering from GZSCET,Bathinda,India (2001).She obtained her Master of Technology degree in Computer Science and Enginee-ing,Punjabi University,Patiala ,India (2005).Her research interests include Software Pro-cess Reengineering,Software Engineering,Agile Software Development,Software Quality Improvement in Small

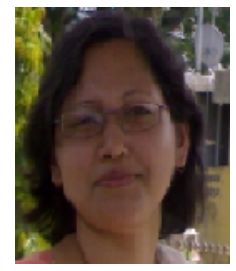
Scale Enterprises,Software Reuse,Software Process-related Custo-Ization and Automation,and Software Process Metrics. 